\title{
Informing Context to Support Adaptive Services
}

\author{
Alexander O'Connor and Vincent Wade \\ Knowledge and Data Engineering Group, \\ Department of Computer Science, \\ Trinity College, \\ Dublin 2, Ireland \\ \{oconnoat, Vincent.Wade\}@cs.tcd.ie
}

\begin{abstract}
A common trend in modern applications is the move towards more mobile, adaptive, customisable software. The evolution of software from static, invariant tools for narrow portions of a task to adaptive, open interaction frameworks is embodied in the use of a variety of technologies for creating a reconfigurable application. Perhaps the two most important techniques are Adaptive architectures and Ubiquitious Computing. However, many techniques employed merging these two technologies to form the vision of a truly ubiquitous, adaptive environment have so far failed to take full account of the expressive quality of both context and adaptivity. This paper presents a new, semantic interopration-based approach to creating context-informed adaptive applications that make maximum use of the rich content that can be found in both technologies.
\end{abstract}

\section{Introduction}

Adaptive Applications are characterised by their variable behaviour in response to their information models[1]. Adaptivity allows for applicationsto make considerable changes for personalisation and customisation preferences as defined by the user and the content being adapted. This mechanism is extremely powerful in providing tailored presentation of information to the user.

Adaptivity also permits this class of applications to leverage Contextual information very effectively, employing additional axes of information to better inform customisation and personalisation. Many of the current approaches to Contextual support for Adaptivity are, however, focused on a mechanism whereby the application itself does a large proportion of the work by querying external sources of information and integrating the results by treating contextual data as separate and distinct. By placing the responsibility for managing and querying and integrating context on the application, this approach can place extra burden on the application programmer, who must design an integration strategy for Context, and on the application itself which potentially makes the information models larget and more complex.

This paper presents a Context-Informed approach to enriching Adaptive applications. In this architecture, the adaptive application is served by a contextual broker, which enriches the application based on a broader spectrum of concerns, 
and which makes use of a model-oriented approach for indirect querying and direct integration. This approach provides and innovative solution using Topic Maps to manage a semantic interoperation process.

\section{Context}

There are a great many[2] definitions and descriptions of what exactly constitutes context, both in general and for particular application domains. For the purposes of this work, it is most important to be aware that contextual information is knowledge about the user, their environment or their task which is not known to the application being used for the task, but which is available at run-time through other services. This extra knowledge can be recognised by a semantic description offered by services surrounding the user and their application, even though the application itself may not know of such information directly.

The system presented follows the context-informed model: the aware environment informs the application. This means that neither the target application nor the sources need to be aware of each other, and that the Target application need only be designed to know that it has the opportunity to gain some extra information, not what that information might be or how it might be acquired.

The principal challenges associated with context-informed support for adaptive services are:

1. To Identify the information need of the adaptive service.

2. To Identify the possible external sources of information.

3. To Identify matching information in sources and infer on its influence on the adaptive service.

4. To determine how to amend the adaptive service both in terms of data and model.

These challenges comprise a broad solution to the process of enhancing adaptive services. Of particular interest in this work are the third and fourth challenges. The process of determining possible sources of context through exploring the information space is, in itself, a very interesting problem. It is likely one made more difficult if the nature of the information and the process by which it is incorporated is not known.

\section{Architecture}

The Architecture is composed of three tiers: the Target Application, which is the system to be enriched; the Sources of Context, which are services that offer additional information to be combined as context, and the Context Exchange, which brokers and manages the process. In proposing this form of architecure, the roles of each participant are clearly defined: it is the Target Application which is enriched by the integrated content returned by the Context Exchange. The enrichment process is performed using a semantic, model-based exchange. 
The internal model of the Target Application is exposed via a schema, and the schema labels are populated by the current state of the application's knowledge. This can then be compared to the information known to Sources of Context via a joined schema that forms the Information Space.

A number of approaches have been suggested[3] for managing contextual information, and enabling transfers. The semantic interoperation approach, as opposed to a planned or workflow model, is desirable because it natually accounts for the dynamic and composite nature of contextual data, as well as generally giving a better responsiveness to change. Topic Maps represent a lightweight 'index' linking the semantically close elements of the different participant ontologies. This provides a navigable framework for creating an enriched model for the Target Application that can be generated based on the definitive underlying ontologies. This system employs Topic Maps to represent the shared schema between the Target Application and the Sources of Context within the Environment. This allows the Context Exchange to create an expressive shared schema that is still lightweight and composable, without the need for the detailed formality that ontologies require. In addition, the ability of the system to alter the Target Applications models as well as data will be dependant on the design of the Target and the Environment.

\subsection{Enhancing Adaptivity}

The underlying benefit to the Adaptive System is that a Context-informed environment facilitates the leveraging of additional information known by external services with minimal a-priori knowledge on the part of the developer. This assists both the content designer and system developer by enabling them to focus on the core concerns of the adaptive application, the remaining information being handled as context. The developer remains in control of the enrichment process by being responsible for determining how and when models are submitted to the Context Exchange.

This approach to deciding what information to treat context can vary greatly for different usage scenarios, depending on the configuration provided at designtime, as well as the properties of the environment at runtime.

\section{Analysis and Future Work}

Currently, the system is in the early stages of implementation. It is intended to employ the system in trials in both the eLearning and corporate collaboration application domains. These areas provide a spectrum of cognitive depth and application adaptivity useful in measuring the generality of this solution and the nature of the tailoring process required by different systems. As an interim approach, it is proposed initially to investigate the properties of this system in a static, pre-determined service environment.

The topic of semantic matching is, in itself, an area of considerable research interest. There are many challenges associated with the process of linking separate, possibly disjoint perspectives, even with the considerable assistance provided by 
ontologies and other semantic techniques. Physical[4] and semantic[5] techniques provide a basis for locating these links, but it is possible that the best achievable solution, in practice, will be a semi-automatic system where the link list is corrected and improved by the user. This has additional advantages in that it assists in maintaining the scrutability of the overall adaptive system - a feature vital in maintaining user interests[6].

The Degree to which the adaptivity of the Target Application affects how this system can be employed. In particular, the process of amending and integrating data and models will likely require some design-time tailoring, depending on the particular properties of the target application and the sources.

\section{Conclusion}

This paper has presented a model-driven, context-informed framework for supporting adaptive applications. The system is based on the principles of semantic interoperation, which provide for the generation of a lightweight aggregate semantic view of the information space which a user, and their application, occupy.

\section{Acknowledgements}

This work is funded by the Irish Research Council for Science, Engineering and Technology: funded by the National Development Plan. Portions of this work are in collaboration with the IBM Centre for Advanced Studies, Dublin.

\section{References}

1. De Bra, P., Aroyo, L., Chepegin, V.: The next big thing: Adaptive web based systems. Journal of Digital Information (5(247, 2005-05-27))

2. Chen, G., Kotz, D.: A Survey of Context-Aware Mobile Computing Research. Technical Report TR2000-381, Dartmouth College, Computer Science, Hanover, NH (2000)

3. Noy, N.F.: Semantic integration: a survey of ontology-based approaches. SIGMOD Rec. 33(4) (2004) 65-70

4. McGuinness, D.L., Fikes, R., Rice, J., , Wilder, S.: The chimaera ontology environment. In: Proceedings of the Seventeenth National Conference on Artificial Intelligence (AAAI 2000). (2000)

5. O'Sullivan, D., Power, R.: Bridging heterogenous, autonomic, dynamic knowledge at runtime. In: 1st International Workshop on Managing Ubiquitous Communications and Services (MUCS). (2003)

6. Czarkowski, M., Kay, J.: How to give the user a sense of control over the personalization of ah? In De Bra, P.e.a., ed.: AH2003: Workshop on Adaptive Hypermedia and Adaptive Web-Based Systems; Workshop proceedings from the Ninth International Conference on User Modeling. (2003) 